\title{
GLOBAL RAINBOW DOMINATION IN GRAPHS
}

\author{
J. AMJADI, S.M. SHEIKHOLESLAMI, AND L. VOLKMANN
}

Received 27 May, 2014

\begin{abstract}
For a positive integer $k$, a $k$-rainbow dominating function (kRDF) of a graph $G$ is a function $f$ from the vertex set $V(G)$ to the set of all subsets of the set $\{1,2, \ldots, k\}$ such that for any vertex $v \in V(G)$ with $f(v)=\varnothing$, the condition $\bigcup_{u \in N(v)} f(u)=\{1,2, \ldots, k\}$ is fulfilled, where $N(v)$ is the neighborhood of $v$. The weight of a $\operatorname{kRDF} f$ is the value $\omega(f)=$ $\sum_{v \in V}|f(v)|$. A kRDF $f$ is called a global k-rainbow dominating function (GkRDF) if $f$ is also a kRDF of the complement $\bar{G}$ of $G$. The global $k$-rainbow domination number of $G$, denoted by $\gamma_{g r k}(G)$, is the minimum weight of a GkRDF on $G$. In this paper, we initiate the study of the global $k$-rainbow domination number and we establish some sharp bounds for it.
\end{abstract}

2010 Mathematics Subject Classification: 05 C69

Keywords: $k$-rainbow dominating function, $k$-rainbow domination number, global $k$-rainbow dominating function, global $k$-rainbow domination number

\section{INTRODUCTION}

In this paper, $G$ is a simple graph with vertex set $V(G)$ and edge set $E(G)$ (briefly $V, E)$. The order $|V|$ of $G$ is denoted by $n=n(G)$. Denote by $K_{n}$ the complete graph, by $C_{n}$ the cycle and by $P_{n}$ the path of order $n$, respectively. For every vertex $v \in V(G)$, the open neighborhood $N_{G}(v)=N(v)$ is the set $\{u \in V(G) \mid u v \in E(G)\}$ and its closed neighborhood is the set $N_{G}[v]=N[v]=N(v) \cup\{v\}$. The degree of a vertex $v \in V$ is $\operatorname{deg}_{G}(v)=\operatorname{deg}(v)=|N(v)|$. The open neighborhood of a set $S \subseteq V$ is the set $N_{G}(S)=N(S)=\cup_{v \in S} N(v)$, and the closed neighborhood of $S$ is the set $N_{G}[S]=N[S]=N(S) \cup S$. The minimum and maximum degrees of $G$ are respectively denoted by $\delta(G)=\delta$ and $\Delta(G)=\Delta$. A leaf of a graph is a vertex of degree 1, a support vertex is a vertex adjacent to a leaf and a strong support vertex is a vertex adjacent to at least two leaves. For a vertex $v$ in a rooted tree $T$, let $C(v)$ denote the set of children of $v$. Let $D(v)$ denote the set of descendants of $v$ and $D[v]=D(v) \cup\{v\}$. The maximal subtree at $v$ is the subtree of $T$ induced by $D[v]$, and is denoted by $T_{v}$. We use [12,19] for terminology and notation which are not defined here.

A subset $S$ of vertices of $G$ is a dominating set if $N[S]=V$. The domination num$\operatorname{ber} \gamma(G)$ is the minimum cardinality of a dominating set of $G$. A dominating set $S$ of 
$G$ is global dominating set of $G$ if $S$ is a dominating set both of $G$ and $\bar{G}$. The global domination number $\gamma_{g}(G)$ of $G$ is the minimum cardinality of a global dominating set. The global domination number was introduced independently by Brigham and Dutton [7] (the term factor domination number was used) and Sampathkumar [15] and has been studied by several authors (see for example [3,20]). Since then some variants of the global domination parameter, such as connected (total) global domination, global minus domination, and global Roman domination, have been studied $[4,5,10,13]$.

For a positive integer $k$, a $k$-rainbow dominating function (kRDF) of a graph $G$ is a function $f$ from the vertex set $V(G)$ to the set of all subsets of the set $\{1,2, \ldots, k\}$ such that for any vertex $v \in V(G)$ with $f(v)=\varnothing$, the condition $\bigcup_{u \in N(v)} f(u)=$ $\{1,2, \ldots, k\}$ is fulfilled. The weight of a $\operatorname{kRDF} f$ is the value $\omega(f)=\sum_{v \in V}|f(v)|$. The $k$-rainbow domination number of a graph $G$, denoted by $\gamma_{r k}(G)$, is the minimum weight of a kRDF of G. A $\gamma_{r k}(G)$-function is a $k$-rainbow dominating function of $G$ with weight $\gamma_{r k}(G)$. Note that $\gamma_{r 1}(G)$ is the classical domination number $\gamma(G)$. The $k$-rainbow domination number was introduced by Brešar, Henning, and Rall [6] and has been studied by several authors (see for example [1, 2, 8, 9, 11, 14, 1618]). A 2-rainbow dominating function (briefly, rainbow dominating function) $f$ : $V \longrightarrow \mathcal{P}(\{1,2\})$ can be represented by the ordered partition $\left(V_{0}, V_{1}, V_{2}, V_{1,2}\right)$ (or $\left(V_{0}^{f}, V_{1}^{f}, V_{2}^{f}, V_{1,2}^{f}\right)$ to refer $\left.f\right)$ of $V$, where $V_{0}=\{v \in V \mid f(v)=\varnothing\}, V_{1}=\{v \in$ $V \mid f(v)=\{1\}\}, V_{2}=\{v \in V \mid f(v)=\{2\}\}$ and $V_{1,2}=\{v \in V \mid f(v)=\{1,2\}\}$. In this representation, its weight is $\omega(f)=\left|V_{1}\right|+\left|V_{2}\right|+2\left|V_{1,2}\right|$.

A kRDF $f$ is called a global $k$-rainbow dominating function (GkRDF) if $f$ is also a kRDF of the complement $\bar{G}$ of $G$. The global k-rainbow domination number of $G$, denoted by $\gamma_{g r k}(G)$, is the minimum weight of a GkRDF on $G$. A $\gamma_{g r k}(G)$-function is a GkRDF of $G$ with weight $\gamma_{g r k}(G)$. Since every global $k$-rainbow dominating function $f$ of $G$ is a kRDF of $G$ and $\bar{G}$, and assigning 1 to the vertices with nonempty label under $f$ is a global dominating set of $G$, and since assigning $\{1,2, \ldots, k\}$ to the vertices of a global dominating set yields a GkRDF, we deduce that

$$
\max \left\{\gamma_{g}(G), \gamma_{r k}(G), \gamma_{r k}(\bar{G})\right\} \leq \gamma_{g r k}(G) \leq k \gamma_{g}(G) .
$$

We note that the global $k$-rainbow domination number can differ significantly from the $k$-rainbow domination number. For example, for $n \geq k+1, \gamma_{r k}\left(K_{n}\right)=k$ and $\gamma_{g r k}\left(K_{n}\right)=n$.

Our purpose in this paper is to initiate the study of the global $k$-rainbow domination number in graphs. We study basic properties of the global $k$-rainbow domination number and we establish some bounds for it.

We make use of the following results in this paper.

Theorem A ([14]). For any graph $G$ of order $n$ and maximum degree $\Delta(G) \geq 1$, $\gamma_{r k}(G) \geq \frac{k n}{\Delta(G)+k}$. 
Theorem B ([6]). For $n \geq 1$,

$$
\gamma_{r 2}\left(P_{n}\right)=\left\lfloor\frac{n}{2}\right\rfloor+1
$$

Theorem C ([6]). For $n \geq 3$,

$$
\gamma_{r 2}\left(C_{n}\right)=\left\lfloor\frac{n}{2}\right\rfloor+\left\lceil\frac{n}{4}\right\rceil-\left\lfloor\frac{n}{4}\right\rfloor .
$$

Theorem D ([1]). If $G$ is a graph of order $n$, then $\gamma_{r k}(G) \leq n-\Delta(G)+k-1$.

Theorem $\mathbf{E}$ ([9]). Let $G$ be a connected graph. If there is a path $v_{3} v_{2} v_{1}$ in $G$ with $\operatorname{deg}\left(v_{2}\right)=2$ and $\operatorname{deg}\left(v_{1}\right)=1$, then $G$ has a $\gamma_{r 2}(G)$-function $f$ such that $f\left(v_{1}\right)=$ $\{1\}$, and $2 \in f\left(v_{3}\right)$.

Since the function $f$ defined by $f(v)=\{1\}$ for each $v \in V(G)$ is a GkRDF of a graph $G$, we have the first part of the following observation. The second part is easy to see and therefore its proof is omitted.

Observation 1. If $G$ is a graph of order $n$, then $\gamma_{g r k}(G) \leq n$. Furthermore, if $1 \leq n \leq 4$, then $\gamma_{g r k}(G)=n$.

$$
\text { 2. GRAPHS WITH } \gamma_{r k}(G)=\gamma_{g r k}(G)
$$

In this section we provide some sufficient conditions for a graph to satisfy $\gamma_{r k}(G)=$ $\gamma_{g r k}(G)$.

Proposition 1. If $G$ is a disconnected graph with at least two components of order at least $k$, then

$$
\gamma_{g r k}(G)=\gamma_{r k}(G) \text {. }
$$

Proof. Let $G_{1}, G_{2}, \ldots, G_{k}$ be the components of $G$. Assume, without loss of generality, that $\left|V\left(G_{i}\right)\right| \geq k$ for $i=1,2$. Let $f$ be a $\gamma_{r k}(G)$-function. Obviously, $\sum_{v \in V\left(G_{i}\right)}|f(v)| \geq k$ for $i=1$,2. If $f(x)=\varnothing$ for some $x \in V\left(G_{i}\right)$, then clearly $\bigcup_{v \in V\left(G_{i}\right)} f(v)=\{1,2, \ldots, k\}$, otherwise we may assume $\bigcup_{v \in V\left(G_{i}\right)} f(v)=$ $\{1,2, \ldots, k\}$ for $i=1,2$ because $\left|V\left(G_{1}\right)\right| \geq k$ and $\left|V\left(G_{2}\right)\right| \geq k$. Then $f$ is a GkRDF of $G$ and hence $\gamma_{g r k}(G) \leq \gamma_{r k}(G)$. Now the result follows from (1.1).

According to Proposition 1, if $G$ is the disjoint union of two copies of the complete graph $K_{n}(n \geq k)$, then $\gamma_{g r k}(G)=\gamma_{r k}(G)$.

Proposition 2. If $G$ is a disconnected graph with $r \geq 2$ components $G_{1}, G_{2}, \ldots$, $G_{r}$ of order at most $k-1$ such that $\sum_{i=1}^{r}\left|V\left(G_{i}\right)\right| \geq k$, then

$$
\gamma_{g r k}(G)=\gamma_{r k}(G) \text {. }
$$

Proof. Assume that $\bigcup_{i=1}^{r} V\left(G_{i}\right)=\left\{v_{1}, v_{2}, \ldots, v_{s}\right\}$, and let $f$ be a $\gamma_{r k}(G)$-function. Then clearly $f\left(v_{i}\right) \neq \varnothing$ for each $i$. Define $g: V(G) \longrightarrow \mathcal{P}(\{1,2, \ldots, k\})$ by $g\left(v_{i}\right)=$ $\{k-i-1\}$ for $1 \leq i \leq k-1, g\left(v_{i}\right)=\{1\}$ for $i=k, k+1, \ldots, s$ and $g(x)=f(x)$ 
for $x \in V(G)-\left\{v_{1}, v_{2}, \ldots, v_{s}\right\}$. Then obviously $g$ is a GkRDF of $G$ of weight $\omega(g)=\gamma_{r k}(G)$ and the proof is complete.

According to Proposition 2, if $\mathrm{G}$ is the disjoint union of $k$ copies of $K_{1}$ and a copy of the complete graph $K_{n}(n \geq k)$, then $\gamma_{g r k}(G)=\gamma_{r k}(G)$.

Theorem 1. For any connected graph $G$ with $\operatorname{radius} \operatorname{rad}(G) \geq 4, \gamma_{g r 2}(G)=$ $\gamma_{r 2}(G)$.

Proof. Let $f=\left(V_{0}, V_{1}, V_{2}, V_{1,2}\right)$ be a $\gamma_{r 2}(G)$-function such that $\left|V_{1,2}\right|$ is maximum. We show that $f$ is a G2RDF of $G$. Suppose to the contrary that $f$ is not a 2 RDF of $\bar{G}$. Then there exists a vertex $v \in V_{0}$ such that $V_{1,2} \subseteq N(v)$ and either $V_{1} \subseteq N(v)$ or $V_{2} \subseteq N(v)$. Assume, without loss of generality, that $V_{1} \subseteq N(v)$. Let $u$ be an arbitrary vertex in $V(G)$. If $u \in V_{1} \cup V_{1,2}$, then $d(u, v)=1$. If $u \in V_{0}$, then $u$ and $v$ have a common neighbor in $V_{1}$ or $V_{1,2}$ implying that $d(u, v) \leq 2$. Let $u \in V_{2}$. If $u$ has a neighbor in $V_{1} \cup V_{1,2}$, then $d(u, v) \leq 2$ as above. If $u$ has a neighbor $w$ in $V_{0}$, then $d(u, v) \leq d(u, w)+d(w, v) \leq 3$. Otherwise, since $G$ is connected, $u$ has a neighbor $x$ in $V_{2}$. Then the function $g$ defined by $g(u)=\varnothing, g(x)=\{1,2\}$ and $g(y)=f(y)$ for $y \in V(G)-\{u, x\}$, is a $\gamma_{r 2}(G)$-function which contradicts the choice of $f$. Thus $f$ is a G2RDF of $G$ and the proof is complete.

Corollary 1. Let $G$ be a connected graph of diameter $\operatorname{diam}(G) \geq 7$. Then

$$
\gamma_{g r 2}(G)=\gamma_{r 2}(G) \text {. }
$$

The next results is an immediate consequence of Theorems B, C and 1.

Corollary 2. For $n \geq 8$,

$$
\gamma_{g r 2}\left(P_{n}\right)=\left\lfloor\frac{n}{2}\right\rfloor+1
$$

Corollary 3. For $n \geq 8$,

$$
\gamma_{g r 2}\left(C_{n}\right)=\left\lfloor\frac{n}{2}\right\rfloor+\left\lceil\frac{n}{4}\right\rceil-\left\lfloor\frac{n}{4}\right\rfloor .
$$

\section{BOUNDS ON THE GLOBAL $k$-RAINBOW DOMINATION NUMBER}

In this section we present some sharp lower and upper bounds on $\gamma_{g r k}(G)$.

Proposition 3. For any integer $k \geq 2$ and any graph $G$ of order $n \geq 2 k$,

$$
\gamma_{g r k}(G) \geq 2 k \text {. }
$$

Proof. Let $f$ be a $\gamma_{g r k}(G)$-function, and let $V_{0}=\{v \in V(G) \mid f(v)=\varnothing\}$. If $V_{0}=\varnothing$, then $\gamma_{g r k}(G)=n \geq 2 k$. Let $V_{0} \neq \varnothing$ and $v \in V_{0}$. Then $\bigcup_{x \in N_{G}(v)} f(x)=$ $\{1,2, \ldots, k\}$ and $\bigcup_{x \in N_{\bar{G}}(v)} f(x)=\{1,2, \ldots, k\}$. Since $N_{G}(v) \cap N_{\bar{G}}(v)=\varnothing$, we obtain $\gamma_{g r k}(G)=\omega(f) \geq 2 k$, as desired. 
This bound is sharp for the disjoint union of two copies of the complete graph $K_{n}(n \geq k+1)$.

Proposition 4. For any graph $G$ of order $n \geq 4, \gamma_{g r 2}(G)=4$ if and only if of $G$ satisfies one of the following properties.

(i) $n=4$,

(ii) there exist two vertices $u$ and $v$ in $G$ such that $N(u) \cap N(v)=\varnothing$ and $N[u] \cup$ $N[v]=V$,

(iii) there exist three distinct vertices $u, v, w$ in $G$ such that $N(u) \cap(N(v) \cup N(w))=$ $\varnothing$ and $N(u) \cup(N(v) \cap N(w))=V-\{u, v, w\}$,

(iv) there exist four distinct vertices $u, v, w, x$ in $G$ such that $(N(u) \cap N(v)) \backslash\{w, x\}=$ $\varnothing,(N(w) \cap N(x)) \backslash\{u, v\}=\varnothing,(N[u] \cup N[v]) \backslash\{w, x\}=V-\{w, x\}$ and $(N[w] \cup$ $N[x]) \backslash\{u, v\}=V-\{u, v\}$.

Proof. If $n=4$, then it is clear that $\gamma_{g r 2}(G)=4$. Let $n \geq 5$. If (ii) holds, then the function $f: V \longrightarrow \mathcal{P}(\{1,2\})$ defined by $f(u)=f(v)=\{1,2\}$ and $f(z)=\varnothing$ for $z \in V(G)-\{u, v\}$, is a 2 RDF of $G$ and $\bar{G}$ which yields $\gamma_{g r 2}(G)=4$ by Proposition 3 . If (iii) holds, then the function $f: V \longrightarrow \mathcal{P}(\{1,2\})$ defined by $f(u)=\{1,2\}, f(v)=$ $\{1\}, f(w)=\{2\}$ and $f(z)=\varnothing$ for $z \in V(G)-\{u, v, w\}$, is a $2 \mathrm{RDF}$ of $G$ and $\bar{G}$ which yields $\gamma_{g r 2}(G)=4$ again. Let (iv) hold. Then the function $f: V \longrightarrow \mathcal{P}(\{1,2\})$ defined by $f(u)=f(v)=\{1\}, f(w)=f(x)=\{2\}$ and $f(z)=\varnothing$ for $z \in V(G)-$ $\{u, v, x, w\}$, is a $2 \mathrm{RDF}$ of $G$ and $\bar{G}$. This implies that $\gamma_{g r 2}(G)=4$.

Conversely, Let $\gamma_{g r 2}(G)=4$ and let $f=\left(V_{0}, V_{1}, V_{2}, V_{1,2}\right)$ be a $\gamma_{g r 2}(G)$-function such that $\left|V_{1,2}\right|$ is maximum. We consider three cases.

Case 1. $\left|V_{1,2}\right|=2$.

Let $V_{1,2}=\{u, v\}$. Then $V_{0}=V(G)-\{u, v\}$. Since $f$ is a G2RDF, each vertex in $w \in V(G)-\{u, v\}$ must be adjacent to a vertex in $\{u, v\}$ in both $G$ and $\bar{G}$. It follows that $N[u] \cup N[v]=V$ and $N(u) \cap N(v)=\varnothing$, i.e. $G$ satisfies (ii).

Case 2. $\left|V_{1,2}\right|=1$.

Then $\left|V_{1}\right|=\left|V_{2}\right|=1$. Let $V_{1,2}=\{u\}, V_{1}=\{v\}$ and $V_{2}=\{w\}$. Hence $V_{0}=V(G)-$ $\{u, v, w\}$. Every vertex of $w \in V(G)-\{u, v, w\}$ must be adjacent to $u$ or both of $v, w$ in $G$ and $\bar{G}$ because $f$ is a 2 RDF of $G$ and $\bar{G}$. This yields $N(u) \cap(N(v) \cup N(w))=$ $\varnothing$ and $N(u) \cup(N(v) \cap N(w))=V-\{u, v, w\}$. Thus $G$ satisfies (iii) in this case.

Case 3. $\left|V_{1,2}\right|=0$.

If $V_{0}=\varnothing$, then $V_{1} \cup V_{2}=V(G)$ which implies that $4=\gamma_{g r 2}(G)=\left|V_{1} \cup V_{2}\right|=n$, i.e. $G$ satisfies (i). Now assume that $V_{0} \neq \varnothing$ and let $z \in V_{0}$. Since $f$ is a $2 \operatorname{RDF}$ of $G$ and $\bar{G}, \bigcup_{v \in N_{G}(z)} f(v)=\{1,2\}$ and $\bigcup_{v \in N_{\bar{G}}(z)} f(v)=\{1,2\}$. Assume that $u, w \in N_{G}(z)$ and $x, v \in N_{\bar{G}}(z)$ such that $f(u)=f(v)=\{1\}$ and $f(w)=f(x)=\{2\}$. Since $f$ is a G2RDF, each vertex in $V(G)-\{u, v, w, x\}$ must be adjacent to a vertex in $\{u, v\}$ and a vertex in $\{w, x\}$ in $G$ and $\bar{G}$. It follows that $(N(u) \cap N(v)) \backslash\{w, x\}=$ $\varnothing,(N(w) \cap N(x)) \backslash\{u, v\}=\varnothing,(N[u] \cup N[v]) \backslash\{w, x\}=V-\{w, x\}$ and $(N[w] \cup$ $N[x]) \backslash\{u, v\}=V-\{u, v\}$. Thus $G$ satisfies (iv). This completes the proof. 
Proposition 5. Let $k \geq 2$ be an integer. If the graph $G$ has $r \geq 1$ components $G_{1}, G_{2}, \ldots, G_{r}$ with $\sum_{i=1}^{r}\left|V\left(G_{i}\right)\right| \leq k-1$ then

$$
\gamma_{g r k}(G) \leq \gamma_{r k}(G)+k-\sum_{i=1}^{r}\left|V\left(G_{i}\right)\right| .
$$

Proof. Let $\bigcup_{i=1}^{r} V\left(G_{i}\right)=\left\{v_{1}, v_{2}, \ldots, v_{s}\right\}$, and let $f$ be a $\gamma_{r k}(G)$-function. Clearly, $f\left(v_{i}\right) \neq \varnothing$ for each $i$. Define $g: V(G) \longrightarrow \mathcal{P}(\{1,2, \ldots, k\})$ by $g\left(v_{s}\right)=\{s, s+$ $1, \ldots, k\}, g\left(v_{i}\right)=\{i\}$ for $i=1,2, \ldots, s-1$ and $g(x)=f(x)$ for $x \in V(G)-\left\{v_{1}, v_{2}, \ldots, v_{s}\right\}$. Then obviously $g$ is a GkRDF of $G$ with weight $\omega(g)=$ $\gamma_{r k}(G)+k-s$ and so $\gamma_{g r k}(G) \leq \gamma_{r k}(G)+k-\sum_{i=1}^{r}\left|V\left(G_{i}\right)\right|$.

Let $H$ be the disjoint union of $r \leq k-1$ isolated vertices and a star $K_{1, s}$ with $s \geq k$. Then $\gamma_{r k}(H)=r+k$ and $\gamma_{g r k}(H)=2 k$. This example demonstrates that Proposition 5 is tight.

Proposition 6. Let $G$ be a graph of order $n \geq 4$ and $u, v \in V(G)$. If $u v \notin E(G)$, then

$$
\gamma_{g r k}(G) \leq n-\operatorname{deg}(u)-\operatorname{deg}(v)+2|N(u) \cap N(v)|+2 k-2,
$$

and if $u v \in E(G)$, then

$$
\gamma_{g r k}(G) \leq n-\operatorname{deg}(u)-\operatorname{deg}(v)+2|N(u) \cap N(v)|+2 k .
$$

Proof. Define $f: V(G) \longrightarrow \mathcal{P}(\{1,2, \ldots, k\})$ as follows

$$
f(z)= \begin{cases}\{1,2, \ldots, k\} & \text { if } z \in\{u, v\} \\ \varnothing & \text { if } z \in((N(u) \cup N(v))-\{u, v\}) \backslash(N(u) \cap N(v)) \\ \{1\} & \text { otherwise. }\end{cases}
$$

It is easy to see that $f$ is a GkRDF of $G$ which attains the bound. This completes the proof.

Corollary 4. If $G$ is a connected triangle-free graph of order $n \geq 4$, then

$$
\gamma_{g r k}(G) \leq \min \left\{n-\Delta(G)-\delta(G)+2 k, \gamma_{r k}(G)+2 k-1\right\} .
$$

Proof. By considering a vertex of maximum degree and one of its neighbors, it follows from Proposition 6 that $\gamma_{g r k}(G) \leq n-\Delta(G)-\delta(G)+2 k$. Hence, it is sufficient to show that $\gamma_{g r k}(G) \leq \gamma_{r k}(G)+2 k-1$. If $n \leq \gamma_{r k}(G)+2 k-1$, the result is immediate. Let $n>\gamma_{r k}(G)+2 k-1$ and let $f$ be a $\gamma_{r k}(G)$-function. Then there exists a vertex $u$ such that $f(u)=\varnothing$. Then $u$ has a neighbor $v$ such that $|f(v)| \geq 1$. Define $g: V(G) \longrightarrow \mathcal{P}(\{1,2, \ldots, k\})$ by $g(u)=g(v)=\{1,2, \ldots, k\}$ and $g(x)=$ $f(x)$ otherwise. Clearly, $g$ is a GkRDF of $G$ and hence $\gamma_{g r k}(G) \leq \gamma_{r k}(G)+2 k-1$. This completes the proof. 
Proposition 7. Let $k \geq 2$ be an integer, and let $G$ be a graph of diameter $\operatorname{diam}(G) \geq$ 5. Then

$$
\gamma_{g r k}(G) \leq \gamma_{r k}(G)+2 k-2 .
$$

Proof. If $G$ is disconnected, then the result follows from Propositions 1 and 5 . Henceforth, we assume that $G$ is connected. Let $f$ be a $\gamma_{r k}(G)$-function. Let $v_{1} v_{2} \ldots v_{d}$ be a diametral path in $G$. If $f\left(v_{1}\right)=f\left(v_{d}\right)=\varnothing$, then we have $\bigcup_{x \in N\left(v_{1}\right)} f(x)=\{1,2, \ldots, k\}$ and $\bigcup_{x \in N\left(v_{d}\right)} f(x)=\{1,2, \ldots, k\}$. Since $\operatorname{diam}(G) \geq$ 5 , we have $N\left(v_{1}\right) \cap N\left(v_{d}\right)=\varnothing$. It follows that $f$ is a GkRDF of $G$ and hence $\gamma_{g r k}(G)=\gamma_{r k}(G)$. If $f\left(v_{1}\right) \neq \varnothing$ and $f\left(v_{d}\right) \neq \varnothing$, then the function $g: V \longrightarrow$ $\mathcal{P}(\{1,2, \ldots, k\})$ defined by $g\left(v_{1}\right)=g\left(v_{d}\right)=\{1,2, \ldots, k\}$ and $g(x)=f(x)$ for $x \in$ $V(G)-\left\{v_{1}, v_{d}\right\}$, is a GkRDF of $G$ of weight at most $\omega(f)+2 k-2$ and so $\gamma_{g r k}(G) \leq$ $\gamma_{r k}(G)+2 k-2$. Now let $f\left(v_{1}\right)=\varnothing$ and $f\left(v_{d}\right) \neq \varnothing$ (the case $f\left(v_{1}\right) \neq \varnothing$ and $f\left(v_{d}\right)=\varnothing$ is similar). Define $g: V \longrightarrow \mathcal{P}(\{1,2, \ldots, k\})$ by $g\left(v_{d}\right)=\{1,2, \ldots, k\}$ and $g(x)=f(x)$ for $x \in V(G)-\left\{v_{d}\right\}$. Obviously, $g$ is a GkRDF of $G$ of weight at most $\omega(f)+k-1$ and so $\gamma_{g r k}(G) \leq \gamma_{r k}(G)+k-1$. This completes the proof.

Proposition 8. If $G$ is a graph of diameter 3 or 4 , then

$$
\gamma_{g r k}(G) \leq \gamma_{r k}(G)+2 k
$$

Proof. Let $f$ be a $\gamma_{r k}(G)$-function, and let $u$ and $v$ be two vertices of $G$ such that $d(u, v)=\operatorname{diam}(G)$. Then the function $g: V \longrightarrow \mathcal{P}(\{1,2, \ldots, k\})$ defined by $g(u)=g(v)=\{1,2, \ldots, k\}$ and $g(x)=f(x)$ for $x \in V(G)-\{u, v\}$, is a GkRDF of $G$ and therefore $\gamma_{g r k}(G) \leq \gamma_{r k}(G)+2 k$.

Theorem 2. If $G$ is a graph of order $n \geq 4$ with minimum degree $\delta(G)$, then

$$
\gamma_{g r k}(G) \leq \gamma_{r k}(G)+\delta(G)+k-1 .
$$

This bound is sharp for stars $K_{1, t}(t \geq 2 k-1)$ by Proposition 3 .

Proof. If $G$ is disconnected, then the result follows from Propositions 1 and 5. Therefore we assume that $G$ is connected. Let $u$ be a vertex of minimum degree $\delta(G), f$ be a $\gamma_{r k}(G)$-function and $B=\{x \in N(u) \mid f(x)=\varnothing\}$.

If $f(u)=\varnothing$, then $\bigcup_{v \in N(u)-B} f(v)=\{1,2, \ldots, k\}$. Then obviously the function $g: V(G) \longrightarrow \mathcal{P}(\{1,2, \ldots, k\})$ defined by $g(u)=\{1,2, \ldots, k\}, g(x)=\{1\}$ if $x \in B$ and $g(z)=f(z)$ otherwise, is a GkRDF of $G$ with weight at most $\gamma_{r k}(G)+\delta(G)+$ $k-1$ and hence $\gamma_{g r k}(G) \leq \gamma_{r k}(G)+\delta(G)+k-1$.

Let $|f(u)| \geq 1$. Define $g: V(G) \longrightarrow \mathcal{P}(\{1,2, \ldots, k\})$ by $g(u)=\{1,2, \ldots, k\}$, $g(v)=\{1\}$ if $v \in B$ and $g(z)=f(z)$ for each $z \in V(G)-(B \cup\{u\})$. It is clear that $g$ is a GkRDF of $G$ with weight at most $\gamma_{r k}(G)+\delta(G)+k-1$ and hence $\gamma_{g r k}(G) \leq \gamma_{r k}(G)+\delta(G)+k-1$. This completes the proof. 


\section{GLOBAL RAINBOW DOMINATION NUMBERS OF TREES}

According to Theorem 2, for any tree $T$ of order $n \geq 4$ we have

$$
\gamma_{g r 2}(T) \leq \gamma_{r 2}(T)+2 \text {. }
$$

In this section we characterize all extremal trees attaining equality in (4.1). We begin with some lemmas giving some sufficient conditions for a tree to have global 2rainbow domination number less than $\gamma_{r 2}(T)+2$. As a special case, Corollary 1 and Proposition 3 imply the next results.

Corollary 5. For any tree $T$ with $\operatorname{diam}(T) \geq 7, \gamma_{g r 2}(T)=\gamma_{r 2}(T)$.

Corollary 6. If $T$ is a star of order $n \geq 4$, then $\gamma_{g r 2}(T)=\gamma_{r 2}(T)+2$.

Lemma 1. Let $T$ be a tree. If $T$ has two strong support vertices, then $\gamma_{g r 2}(T) \leq$ $\gamma_{r 2}(T)+1$.

Proof. Let $u$ and $v$ be two strong support vertices of $T$ and let $f$ be a $\gamma_{r 2}(T)$ function. Obviously we may assume that $f(u)=f(v)=\{1,2\}$. Since $T$ is a tree, $u$ and $v$ have at most one common neighbor. If $u$ and $v$ have no common neighbor, then clearly $f$ is a G2RDF of $T$ and hence $\gamma_{g r 2}(T)=\gamma_{r 2}(T)$. If $u$ and $v$ has a common neighbor, say $w$, then the function $g$ defined by $g(w)=f(w) \cup\{1\}$ and $g(x)=f(x)$ otherwise, is a G2RDF of $T$ of weight at most $\gamma_{r_{2}}(T)+1$ and the result follows.

Lemma 2. Let $T$ be a tree. If $\operatorname{diam}(T)=6$, then $\gamma_{g r 2}(T)=\gamma_{r 2}(T)$.

Proof. Let $P=v_{1} v_{2} \ldots v_{7}$ be a diametral path of $T$ and let $f$ be a $\gamma_{r 2}(T)$ function. Root $T$ at $v_{1}$. If $v_{2}$ and $v_{6}$ are strong support vertices, then $f$ is a $\gamma_{g r 2}(T)$ function since $v_{2}$ and $v_{6}$ have no common neighbor. Hence $\gamma_{g r 2}(T)=\gamma_{r 2}(T)$. Assume, without loss of generality, that $\operatorname{deg}\left(v_{2}\right)=2$. By Theorem E, we may assume $f\left(v_{1}\right)=\{1\}$ and $2 \in f\left(v_{3}\right)$. If $v_{6}$ is a strong support vertex, then we can assume $f\left(v_{6}\right)=\{1,2\}$ and clearly $f$ is a G2RDF of $T$ implying that $\gamma_{g r 2}(T)=\gamma_{r_{2}}(T)$. Henceforth, we assume $\operatorname{deg}\left(v_{6}\right)=2$. By Theorem E, we may assume $f\left(v_{7}\right)=\{1\}$ and $2 \in f\left(v_{5}\right)$. Define the function $g$ by $g(v)=\{1\}$ if $v \in V\left(T_{v_{5}}\right)$ and $f(v)=\{2\}$, $g(v)=\{2\}$ if $v \in V\left(T_{v_{5}}\right)$ and $f(v)=\{1\}$ and $g(x)=f(x)$ otherwise. Clearly, $g$ is a G2RDF of $T$ of weight $\gamma_{r 2}(T)$ and hence $\gamma_{g r 2}(T)=\gamma_{r 2}(T)$. This completes the proof.

Lemma 3. Let $T$ be a tree. If $\operatorname{diam}(T)=5$, then $\gamma_{g r 2}(T) \leq \gamma_{r 2}(T)+1$.

Proof. Let $P=v_{1} v_{2} \ldots v_{6}$ be a diametral path of $T$, and let $f$ be a $\gamma_{r 2}(T)$ function. If $v_{2}$ and $v_{5}$ are strong support vertices, then $f$ is a $\gamma_{g r 2}(T)$-function and hence $\gamma_{g r 2}(T)=\gamma_{r_{2}}(T)$. Assume, without loss of generality, that all support vertices adjacent to $v_{4}$ have degree 2 . By Theorem E, we may assume $f\left(v_{6}\right)=\{1\}$ and $2 \in f\left(v_{4}\right)$. Then the function $g$ defined by $g\left(v_{3}\right)=f\left(v_{3}\right) \cup\{1\}$ and $g(x)=f(x)$ otherwise, is a G2RDF of $T$ of weight at most $\gamma_{r 2}(T)+1$ that implies $\gamma_{g r 2}(T) \leq$ $\gamma_{r 2}(T)+1$. 
A subdivision of an edge $u v$ is obtained by removing the edge $u v$, adding a new vertex $w$, and adding edges $u w$ and $w v$. The subdivision graph $S(G)$ is the graph obtained from $G$ by subdividing each edge of $G$. The subdivision $\operatorname{star} S\left(K_{1, t}\right)$ for $t \geq$ 2 , is called a healthy spider. A wounded spider $S_{t}$ is the graph formed by subdividing at most $t-1$ of the edges of a star $K_{1, t}$ for $t \geq 2$. The center of a spider, is the center of the star whose subdivision produced the spider.

Definition 1. For $1 \leq i \leq 2$, let $\mathscr{B}_{i}$ be the family of trees $T$ defined as follows and let $\mathscr{B}=\bigcup_{i=1}^{2} \mathscr{B}_{i}$.

$\mathscr{B}_{1}$ : $T$ is a spider $S_{t}$ for some $t \geq 2$ with exception of stars, wounded spiders $S_{t}(t \geq 3)$ with exactly one wounded leg or wounded spiders $S_{t}(t \geq 3)$ with at least four healthy legs.

$\mathscr{B}_{2}: T$ is obtained from stars $K_{1, r_{1}}, K_{1, r_{2}}, \ldots, K_{1, r_{j}}$ where $r_{k} \geq 3$ for $1 \leq k \leq j$, with centers $y_{1}, y_{2}, \ldots, y_{j}(j \geq 2)$ by adding a new vertex $x$ and joining $x$ to all vertices $y_{j}$ and adding at most one pendant edge at $x$.

Lemma 4. Let $T$ be a tree. If $\operatorname{diam}(T)=4$, then $\gamma_{g r 2}(T) \leq \gamma_{r_{2}}(T)+1$ and equality holds if and only if $T \in \mathscr{B}$.

Proof. Let $\operatorname{diam}(T)=4$ and let $P=v_{1} v_{2} v_{3} v_{4} v_{5}$ be a diametral path of $T$. Let $f$ be a $\gamma_{r 2}(T)$-function. Consider the following cases.

Case 1. $\operatorname{deg}\left(v_{2}\right)=3$.

Suppose $u, v_{1}$ are the leaves adjacent to $v_{2}$. Then we can assume that $f\left(v_{2}\right)=\{1,2\}$. If $\operatorname{deg}\left(v_{4}\right) \geq 3$, then we may assume $f\left(v_{4}\right)=\{1,2\}$ and if $\operatorname{deg}\left(v_{4}\right)=2$ then by Theorem E we can assume $f\left(v_{5}\right)=\{1\}$ and $2 \in f\left(v_{3}\right)$. Define $g: V(T) \rightarrow \mathcal{P}(\{1,2\})$ by $g\left(v_{1}\right)=\{1\}, g(u)=\{2\}, g\left(v_{2}\right)=\varnothing$ and $g(x)=f(x)$ otherwise. Obviously $g$ is a G2RDF of $T$ of weight $\gamma_{r_{2}}(T)$ and hence $\gamma_{g r 2}(T)=\gamma_{r 2}(T)$.

By Case 1 , we may assume that all support vertices adjacent to $v_{3}$ have degree different from 3.

Case 2. $\operatorname{deg}\left(v_{2}\right)>3$.

Then $f\left(v_{2}\right)=\{1,2\}$. If $\operatorname{deg}\left(v_{4}\right)=2$, then by Theorem E we may assume $f\left(v_{5}\right)=\{1\}$ and $2 \in f\left(v_{3}\right)$, and clearly $f$ is a G2RDF of $T$ and hence $\gamma_{g r 2}(T)=\gamma_{r 2}(T)$. So we assume that each support vertex adjacent to $v_{3}$ has degree at least 4 . If $v_{3}$ is a strong support vertex, then $f\left(v_{3}\right)=\{1,2\}$ and clearly $f$ is a G2RDF of $T$ and hence $\gamma_{g r 2}(T)=\gamma_{r 2}(T)$. Let $v_{3}$ be not a strong support vertex. Then $T \in \mathscr{B}_{2}$ and $T$ has at most two $\gamma_{r 2}(T)$-functions which none of them is G2RDF of $T$ and hence $\gamma_{g r 2}(T) \geq \gamma_{r 2}(T)+1$. On the other hand, the function $g$ defined by $g\left(v_{3}\right)=\{1\}$ and $g(x)=f(x)$ otherwise is a G2RDF of $T$ of weight $\gamma_{r 2}(T)+1$ implying that $\gamma_{g r 2}(T)=\gamma_{r 2}(T)+1$.

By Cases 1 and 2, we may assume that all support vertices adjacent to $v_{3}$ have degree 2. Thus $T$ is a spider of diameter 4 . If $T$ is a wounded spiders $S_{t}(t \geq 3)$ with exactly one wounded leg, then the function $g$ that assigns $\varnothing$ to all support vertices 
of $T$ with exception of the center of spider, $\{1\}$ to the center of spider and the leaf adjacent to the center of spider, and $\{2\}$ to the other leaves, is a G2RDF of $T$ of weight $\gamma_{r 2}(T)$ implying that $\gamma_{g r 2}(T)=\gamma_{r 2}(T)$. Now $T$ is a wounded spider $S_{t}(t \geq 3)$ with at least four healthy legs. Suppose $x$ is the center of $T$ and $u_{1}, u_{2}, u_{3}, u_{4}$ are leaves at distance two from $x$. Then the function $g$ that assigns $\{1,2\}$ to $x, \varnothing$ to all support vertices of $T,\{1\}$ to $u_{1}, u_{2}$, and $\{2\}$ to the other leaves, is a G2RDF of $T$ of weight $\gamma_{r 2}(T)$ implying that $\gamma_{g r 2}(T)=\gamma_{r 2}(T)$. Finally let $T$ be a spider that is not a wounded spider $S_{t}(t \geq 3)$ with exactly one wounded leg or a wounded spider $S_{t}(t \geq 3)$ with at least four healthy legs, that is $T \in \mathscr{B}_{1}$. It is easy to see that in this case $\gamma_{g r 2}(T)=\gamma_{r 2}(T)+1$ and the proof is complete.

For $p, q \geq 1$, a double star $D S(p, q)$ is a tree with exactly two vertices that are not leaves, with one adjacent to $p$ leaves and the other to $q$ leaves.

Lemma 5. Let $T$ be a tree. If $\operatorname{diam}(T)=3$, then $\gamma_{g r 2}(T) \leq \gamma_{r 2}(T)+1$ and equality holds if and only if $T=D S(p, q)$ with $q \geq p=1$.

Proof. Let $\operatorname{diam}(T)=3$. Then $T$ is a double star $D S(p, q)$ with $q \geq p \geq 1$. Let $u, v$ be the vertices of $T$ of degree $p$ and $q$, respectively. If $p \geq 2$, then $u, v$ are strong support vertices with no common neighbor and it follows from the proof of Lemma 1 that $\gamma_{g r 2}(T)=\gamma_{r 2}(T)$. Henceforth, assume $p=1$. If $q=1$, then $T=P_{4}$ and clearly $\gamma_{g r 2}(T)=\gamma_{r 2}(T)+1$. Let $q \geq 2$ and $u^{\prime}$ be the leaf adjacent to $u$. Then $T$ has exactly two $\gamma_{r 2}(T)$-functions $f_{i}(i=1,2)$ defined by $f_{i}(v)=\{1,2\}, f_{i}\left(u^{\prime}\right)=\{i\}$ and $f_{i}(x)=\varnothing$ otherwise. Obviously, none of $f_{1}$ or $f_{2}$ is not a G2RDF of $T$ and also the function $g$ defined by $g(u)=\{1\}$ and $g(x)=f_{1}(x)$ for $x \in V(T)-\{u\}$ is a G2RDF of $T$ that yields $\gamma_{g r 2}(T) \geq \gamma_{r 2}(T)+1$.

The next theorem is an immediate consequence of (4.1), Corollaries 5, 6 and Lemmas 2, 3, 4, 5 .

Theorem 3. Let $T$ be a tree of order $n \geq 4$. Then $\gamma_{g r 2}(T)=\gamma_{r 2}(T)+2$ if and only if $T$ is the star $K_{1, t}$ for some $t \geq 3$.

\section{ACKNOWLEDGEMENT}

The authors would like to thank anonymous referees for their remarks and suggestions that helped improve the manuscript.

\section{REFERENCES}

[1] J. Amjadi, A. Bahremandpour, S. M. Sheikholeslami, and L. Volkmann, "The rainbow domination number of a digraph," Kragujevac J. Math., vol. 37, no. 2, pp. 257-268, 2013.

[2] J. Amjadi, N. Mohammadi, S. M. Sheikholeslami, and L. Volkmann, "The $k$-rainbow bondage number of a digraph,” Discuss. Math. Graph Theory, vol. 35, no. 2, pp. 261-270, 2015, doi: 10.7151/dmgt.1797.

[3] S. Arumugam, K. Karuppasamy, and I. S. Hamid, "Fractional global domination in graphs," Discuss. Math. Graph Theory, vol. 30, no. 1, pp. 33-44, 2010, doi: 10.7151/dmgt.1474. 
[4] M. Atapour, S. Norouzian, and S. M. Sheikholeslami, "Global minus domination in graphs," Trans. Comb., vol. 3, no. 2, pp. 35-44, 2014.

[5] M. Atapour, S. M. Sheikholeslami, and L. Volkmann, "Global Roman domination in trees," Graphs Combin., vol. 31, no. 4, pp. 813-825, 2015, doi: 0.1007/s00373-014-1415-3.

[6] B. Brešar and T. K. Šumenjak, "On the 2-rainbow domination in graphs," Discrete Appl. Math., vol. 155, no. 17, pp. 2394-2400, 2007, doi: 10.1016/j.dam.2007.07.018.

[7] R. C. Brigham and R. D. Dutton, "Factor domination in graphs," Discrete Math., vol. 86, no. 1, pp. 127-136, 1990, doi: 10.1016/0012-365X(90)90355-L.

[8] G. J. Chang, J. Wu, and X. Zhu, "Rainbow domination on trees," Discrete Appl. Math., vol. 158, no. 1, pp. 8-12, 2010, doi: 10.1016/j.dam.2009.08.010.

[9] N. Dehgardi, S. M. Sheikholeslami, and L. Volkmann, "The rainbow domination subdivision numbers of graphs," Mat. Vesnik, vol. 67, no. 2, pp. 102-114, 2015.

[10] D. Delic and C. Wang, "The global connected domination in graphs," Ars Combin., vol. 114, pp. 105-110, 2014.

[11] M. Falahat, S. M. Sheikholeslami, and L. Volkmann, "New bounds on the rainbow domination subdivision number," Filomat, vol. 28, no. 3, pp. 615-622, 2014, doi: 0.2298/FIL1403615F.

[12] T. W. Haynes, S. T. Hedetniemi, and P. J. Slater, Fundamentals of domination in graphs. New York: Marcel Dekker, Inc., 1998.

[13] V. R. Kulli and B. Janakiram, "The total global domination number of a graph," India J. Pure Appl. Math., vol. 27, pp. 537-542, 1996.

[14] D. Meierling, S. M. Sheikholeslami, and L. Volkmann, "Nordhaus-Gaddum bounds on the krainbow domatic number of a graph," Applied Math. Lett., vol. 24, no. 10, pp. 1758-1761, 2011, doi: 10.1016/j.aml.2011.04.046.

[15] E. Sampathkumar, "The global domination number of a graph," J. Math. Phys. Sci., vol. 23, no. 5, pp. 377-385, 1989.

[16] S. M. Sheikholeslami and L. Volkmann, "The k-rainbow domatic number of a graph," Discuss. Math. Graph Theory, vol. 32, no. 1, pp. 129-140, 2012, doi: 10.7151/dmgt.1591.

[17] S. M. Sheikholeslami and L. Volkmann, "The $k$-rainbow domination and domatic numbers of digraphs,” Kyungpook Math. J., vol. 56, no. 1, pp. 69-81, 2016.

[18] C. Tong, X. Lin, Y. Yang, and M. Luo, "2-rainbow domination of generalized petersen graphs p (n, 2)," Discrete Appl. Math., vol. 157, no. 8, pp. 1932-1937, 2009, doi: 10.1016/j.dam.2009.01.020.

[19] D. B. West, Introduction to graph theory. Prentice hall Upper Saddle River, 2001.

[20] V.Zverovich and A. Poghosyan, "On Roman, global and restrained domination in graphs," Graphs Combin., vol. 27, no. 5, pp. 755-768, 2011, doi: 10.1007/s00373-010-0992-z.

Authors' addresses

J. Amjadi

Azarbaijan Shahid Madani University, Department of Mathematics, Tabriz, I.R. Iran

E-mail address: j-amjadi@azaruniv.edu

\section{S.M. Sheikholeslami}

Azarbaijan Shahid Madani University, Department of Mathematics, Tabriz, I.R. Iran

E-mail address: s.m.sheikholeslami@azaruniv.edu

\section{Volkmann}

RWTH Aachen University, Lehrstuhl II für Mathematik, 52056, Aachen, Germany

E-mail address: volkmemath2.rwth-aachen. de 